\title{
La implementación de la lista de verificación para una cirugía segura y su impacto en la morbimortalidad
}

\author{
The implementation of the checklist for safe surgery and its impact \\ on morbidity and mortality \\ Elena López-Gavito, * Josafat Yair Arroyo-Aparicio, ** \\ Afranía Angelina Zamora-Lizárraga,*** Arantxa Montalvo-López Gavito****
}

Palabras clave:

Cirugía Segura Salva

Vidas, complicaciones postquirúrgicas, lista de verificación.

Key words:

"Safe surgery saves lives", surgical complications, checklist.

* Cirujana General. Hospital Sharp, Mazatlán, Sinaloa.

*** Médico General. Hospital Naval de Mazatlán, Sinaloa.

*** Médica

Anestesióloga. Hospital

Naval de Mazatlán,

Sinaloa.

**** Pasante de

Medicina, Universidad

Anáhuac Norte.

Recibido: 31/03/2016

Aceptado: 19/07/2016

\section{RESUMEN}

En los países industrializados, aproximadamente entre tres y $16 \%$ de los procedimientos quirúrgicos que requieren de hospitalización presentan complicaciones que generan discapacidad permanente o tasas de mortalidad en el 0.4$0.8 \%$ (en cirugías mayores, del 0.5-5\%). La Secretaría de Marina-Armada de México ha puesto en marcha el programa Cirugía Segura Salva Vidas, en el cual promueve la formulación de nuevas políticas y programas con el fin de reducir los eventos adversos derivados de la práctica quirúrgica. Material y métodos: Estudio de cohortes que compara la incidencia de eventos adversos y mortalidad tras aplicar una estrategia de seguridad en el paciente quirúrgico. Se implementó la lista de verificación a 255 individuos llevados a cirugía no ambulatoria, programados electivamente o de urgencia en el Hospital Naval de Mazatlán en el periodo del 01 de enero al 30 de junio de 2010, así como a 280 en el periodo del 01 de enero al 30 de junio de 2012, con un total de 535 sujetos incluidos. Resultados: Las complicaciones disminuyeron de $11.4 \%$ a $4.3 \%(\mathrm{p}=0.02)$, con un RR de (0.377), protegiendo contra complicaciones potencialmente prevenibles en un $66.3 \%$. El tipo de complicaciones donde se encontró una mayor relevancia estadística fueron la infección postquirúrgica y la hemorragia mayor, con $77 \%(\mathrm{p}=0.03)$ y $59.7 \%(\mathrm{p}$ $=0.02$ ) menos complicaciones. Conclusión: Se observó una reducción de más del $60 \%$ de las complicaciones tras aplicar la lista de verificación de cirugía segura, con una efectividad comparable a la reportada en el resto de los estudios realizados en hospitales a nivel internacional.

\section{ABSTRACT}

Approximately between three to $16 \%$ of surgical procedures requiring admission in industrialized countries have complications, generating permanent disability or mortality rates of in 0.4 to $0.8 \%$ and 0.5 to $5 \%$ mortality in major surgeries. The Mexican Secretariat of the Navy and Armed Forces has launched the "Safe Surgery Saves Lives" program, which promotes the development of new policies and programs in order to reduce adverse events resulting from surgical practice. Material and methods: Cohort study comparing the incidence of adverse events and mortality after applying a security strategy in the surgical patient. Two hundred and fifty-five patients undergoing non-ambulatory surgery were studied, scheduled electively or urgently in the period from January $1^{\text {st }}$ to June 30, 2010, and 280 in the period from January 1 to June 30, 2012, at the Naval Hospital in Mazatlan, totaling 535 patients. Results: Complications decreased from $11.4 \%$ to $4.3 \%(p=0.02)$, with a RR of (0.377), protecting against potentially preventable complications in $66.3 \%$. The type of complications that found a higher statistical significance were postsurgical infection and increased allowable blood loss: $77 \%(p=0.03)$ and $59.7 \%(p=0.02)$ fewer complications. Conclusion: A reduction of more than $60 \%$ of complications was observed after applying the checklist for safe surgery, with comparable effectiveness with other studies in hospitals worldwide.

\section{INTRODUCCIÓN}

$\mathrm{A}$ ctualmente, la cirugía representa un elemento clave de la atención médica integral, con aproximadamente 234 millones de cirugías realizadas mundialmente en el último año. Por ello, las complicaciones quirúrgicas son un problema de salud inminente al representar la tercera causa de muerte a nivel mundial, cuando en su mayoría son potencialmente prevenibles. ${ }^{1,2}$ 
En países industrializados, aproximadamente entre tres y $16 \%$ de los procedimientos quirúrgicos que requieren hospitalización presentan complicaciones importantes que generan discapacidad permanente o tasas de mortalidad de 0.4 a $0.8 \%$ (y de 0.5 a $5 \%$ en cirugías mayores). ${ }^{1,2}$

Se calcula que, cada año, 63 millones de personas se someten a intervenciones quirúrgicas por lesiones traumáticas, otros 10 millones por complicaciones relacionadas con el embarazo y 31 millones más por problemas oncológicos. ${ }^{1,2}$

La Organización Mundial de la Salud (OMS) creó la Alianza Mundial para la Seguridad del Paciente, donde elaboró los retos mundiales en este tema. El programa de seguridad en la cirugía y la implementación de la lista de verificación surgen como propuestas para garantizar que los equipos quirúrgicos de salud adopten de forma sistemática medidas esenciales para minimizar los riesgos evitables que comúnmente ponen en peligro el bienestar y la vida del paciente quirúrgico, además de proporcionar un conjunto sencillo y eficiente de controles prioritarios para mejorar la eficacia del trabajo en equipo, la comunicación, y fomentar la concientización activa de la seguridad del enfermo. ${ }^{2-5}$

La instauración de la lista propuesta por la OMS reduce de forma significativa la tasa de complicaciones del 11 al 7\%, la de mortalidad del 1.5 al $0.8 \%$. Lo más significativo de este estudio es que en esta reducción contribuyeron gran cantidad de centros hospitalarios en diferentes países; es decir, que la lista de verificación es útil en cualquier institución que proporcione atención médica. .,6 $^{5}$

Indudablemente, la experiencia del cirujano, anestesiólogo y equipo quirúrgico son factores cruciales para reducir errores; sin embargo, estos en su mayoría son prevenibles si se instauran y protocolizan las medidas necesarias. La implementación de la lista de verificación de Cirugía Segura Salva Vidas es una iniciativa multifacética y participativa para reducir el daño al paciente mediante una atención quirúrgica más segura. ${ }^{5,6}$ La lista de la OMS pretende garantizar la incorporación de elementos clave para la seguridad en el trabajo que se realiza habitualmente en los quirófanos.
Se entiende que las normas representan un cambio de hábitos en casi todos los entornos, pero su introducción se ha basado en datos científicos y amplios estudios. ${ }^{2-5}$

Los objetivos que persigue todo equipo quirúrgico en los diferentes momentos dentro de la sala de operaciones se condensaron en esta lista de verificación utilizando puntos clave para cerciorarse de que se cumplen las normas de seguridad; esta herramienta permite en unos cuantos minutos subsanar alguna situación que pudiera haberse pasado por alto.

La lista de verificación divide la operación en tres fases, cada una correspondiente a un periodo concreto en el curso normal de una intervención: el periodo anterior a la inducción de la anestesia (entrada), el periodo posterior a la inducción de la anestesia y anterior a la incisión quirúrgica (pausa quirúrgica), y el periodo de cierre de la herida quirúrgica o inmediatamente posterior, pero anterior a la salida del paciente del quirófano (salida). En cada una de las fases, antes de continuar con el procedimiento, se ha de permitir que un coordinador confirme que el equipo ha llevado a cabo sus tareas habituales y anunciar en voz alta la ejecución de cada uno de esos pasos.

Casi todos los pasos serán confirmados verbalmente por el personal que corresponda con el fin de garantizar la realización de las acciones clave. Durante la fase de "entrada”, el coordinador de la lista confirmará verbalmente con el paciente su identidad, el lugar anatómico de la intervención y el procedimiento, así como su consentimiento para ser operado. Verificará visualmente que se ha delimitado el sitio quirúrgico, si procede, y que el individuo tiene colocado un oxímetro de pulso que funciona correctamente. Además, revisará verbalmente con el anestesiólogo el riesgo de hemorragia, dificultades en el acceso a la vía aérea y de reacciones alérgicas que presenta el sujeto, y si se ha llevado a cabo una comprobación de la seguridad del equipo de anestesia. El cirujano debe estar presente en la fase de entrada, ya que puede tener una idea más clara de la hemorragia prevista, las alergias $\mathrm{u}$ otras posibles complicaciones; sin embargo, su presencia no es esencial para completar esta parte.

En la "pausa quirúrgica", el equipo se detendrá justo antes de la incisión cutánea 
para confirmar en voz alta que se va a realizar la operación correcta en el paciente y el sitio correctos; a continuación, los miembros del equipo revisarán verbalmente entre sí, por turnos, los puntos fundamentales de su plan de intervención, utilizando como guía las preguntas de la lista de verificación. Se confirmará si se han administrado antibióticos profilácticos en los 60 minutos anteriores, si es que procede, y si pueden visualizarse adecuadamente los estudios de imagen esenciales.

En la etapa de "salida”, todos los miembros del equipo revisarán la operación llevada a cabo y realizarán el recuento de gasas e instrumentos y el etiquetado de toda muestra biológica obtenida. También examinarán los problemas que pudieran haberse producido en relación con el funcionamiento del instrumental o los equipos, y otros problemas que deban resolverse. Por último, antes de que el paciente salga del quirófano, repasarán los planes y aspectos principales del tratamiento postoperatorio y la recuperación.

Se requiere garantizar el avance en materia de seguridad en la cirugía, por lo que debe ponerse en funcionamiento una vigilancia sistemática que permita evaluar y medir los servicios quirúrgicos. Se ha demostrado que la implementación de la campaña de "la lista de seguridad Cirugía Segura Salva Vidas” se asocia con la reducción de complicaciones postoperatorias, así como de la mortalidad de los pacientes sometidos a algún evento quirúrgico (Figura 1).

Los procedimientos quirúrgicos se han convertido en un componente esencial de la asistencia sanitaria. La Secretaría de MarinaArmada de México, en cumplimiento de su compromiso por la mejora continua en los servicios de salud, ha puesto en marcha el programa Cirugía Segura Salva Vidas, en el cual promueve la formulación de nuevas políticas y programas que traten aspectos sistemáticos

\section{Lista de verificación de la seguridad de la cirugía (1.? edición)}

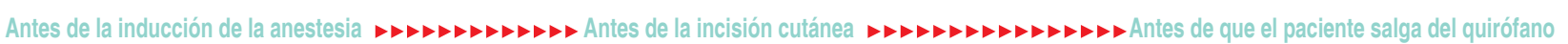

\section{Entrada}

$\square$ El paciente ha confirmado: - Su identidad.

- El sitio quirúrgico.

- El procedimiento.

- Su consentimiento.

$\square$ Demarcación de sitio/no procede.

$\square$ Si ha completado el control de la seguridad de la anestesia.

$\square$ Pulsioxímetro colocado y en funcionamiento.

¿Tiene el paciente: alergias conocidas?

$\square$ No

$\square$ Sí.

¿Vía aérea difícil/riesgo de aspiración?

$\square$ No.

$\square$ Sí, y hay instrumental y equipos/ayuda disponible.

Riesgo de hemorragia $>500 \mathrm{ml}(7 \mathrm{ml} / \mathrm{EG}$ en niños)

$\square$ No.

$\square$ Sí, y se ha provisto la disponibilidad de acceso intravenoso y líquidos adecuados.

\section{Pausa quirúrgica}

$\square$ Confirmar que todos los miembros del equipo se hayan presentado por su nombre y función.

$\square$ Cirujano, anestesista y enfermero, confirmar verbalmente: - La identidad del paciente. - El sitio quirúrgico. - El procedimiento

Previsión de eventos críticos: El cirujano revisa: los pasos críticos e imprevistos, la duración de la operación y la pérdida de sangre prevista.

$\square$ El equipo de anestesia revisa: si el paciente presenta algún problema específico.

$\square$ El equipo de enfermería revisa: si se ha confirmado la esterilidad (con resultados de los indicadores) y si existen dudas o problemas relacionados con el instrumental y los equipos.

¿Se ha administrado profilaxis antibiótica en los últimos 60 minutos?

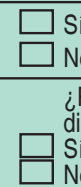
Sí.

No procede.

¿Pueden visualizarse las imágenes diagnósticas esenciales?

Sí.

No procede.

\section{Salida}

El enfermero confirma verbalmente con el equipo:

El nombre del procedimiento realizado.

Que los recuentos de instrumentos, gasas y agujas son correctos (o no proceden)

El etiquetado de las muestras (que figure el nombre del paciente).

$\square$ Si hay problemas que resolver relacionados con el instrumental y los equipos.

$\square$ El cirujano, el anestesista y la enfermera revisan los principales aspectos de la recuperación y el tratamiento del paciente.

Figura 1. Lista de verificación de la seguridad de la cirugía. 
y técnicos, además de sensibilizar la práctica médico-quirúrgica para mejorar la seguridad del paciente. Uno de los programas implementados ha sido la incorporación de la lista de verificación de seguridad en la cirugía, con el fin de reducir los eventos adversos derivados de la práctica quirúrgica.

\section{MATERIAL Y MÉTODOS}

El estudio fue realizado en el Hospital Naval de Mazatlán, Sinaloa, por un equipo multidisciplinario conformado por un cirujano, un anestesiólogo, enfermería, un médico interno de pregrado, así como el personal encargado de control de calidad.

Los efectos de la lista de verificación y los resultados arrojados fueron estudiados mediante un ensayo controlado, tipo cohorte, donde se compararon los resultados antes y después de la implementación de la estrategia de seguridad para el paciente quirúrgico en los periodos correspondientes del primero de enero al 30 de junio de 2010 y del primero de enero al 30 de junio de 2012 en el Hospital Naval de Mazatlán.

El periodo de línea de base de medición fue de seis meses, durante los cuales se documentaron las complicaciones de los pacientes de cualquier género, de todas las edades, derechohabientes y militares que se sometieron a alguna intervención quirúrgica. Se excluyeron las cirugías cardiotorácicas por no realizarse en el Hospital Naval de Mazatlán; las cirugías de tipo ambulatorio no fueron incluidas en el estudio por la dificultad del registro y seguimiento en este tipo de individuos.

El estudio constó de dos etapas. En la primera, se recolectaron los datos de los sujetos intervenidos quirúrgicamente durante el periodo de 2010, antes de la implementación de la lista de verificación de cirugía segura, basándonos en el control diario de cirugías, para el análisis subsecuente del expediente en busca de complicaciones registradas hasta 30 días posteriores a la fecha de intervención quirúrgica; se confirmó vía telefónica con cada paciente. La segunda etapa consistió en la recolección de datos de las personas intervenidas quirúrgicamente en el periodo correspondiente a 2012, en donde el programa de cirugía segura ya se llevaba a cabo en todos los individuos sometidos a algún procedimiento quirúrgico de manera rutinaria; se usó el mismo mecanismo de recolección de datos.

Se estudiaron los casos de 255 sujetos quirúrgicos llevados a cirugía no ambulatoria, programados electivamente o de urgencia en 2010, antes de la aplicación de la lista de verificación en el Hospital Naval de Mazatlán, y 280 pacientes de las mismas características en 2012, ya con el uso de la lista de verificación, lo que nos da un total de 535 individuos incluidos en el estudio.

A partir de la revisión de los expedientes clínicos de cada uno de los sujetos incluidos en la investigación, se elaboró una hoja de recolección de información donde se incluyeron las variables de edad, género, diagnóstico, cirugía programada, cirugía realizada, tipo de cirugía, estatificación de la Sociedad Americana de Anestesiología (ASA), así como las complicaciones o reacciones adversas que se presentaron en la persona intervenida quirúrgicamente, como muerte, hemorragia, infección del sitio quirúrgico, reacciones adversas a medicamentos, iatropatogenia: lesión de la cámara posterior del ojo izquierdo; complicaciones anestésicas, trombóticas y otras, como rechazo al material de osteosíntesis, fallas en el funcionamiento de los equipos, caída del paciente. Aquellas complicaciones que surgieron después del periodo de base de seis meses no se documentaron.

Posteriormente, toda la información recabada en la hoja de recolección fue capturada en una base de datos mediante el uso del programa SPSS versión 19.0.

Se obtuvieron los datos de cada cirugía a través de una hoja de recolección de datos estandarizada por los médicos involucrados, los cuales llevaron un entrenamiento previo de recolección por el investigador para medir adecuadamente y que existieran menos fallas durante el proceso de recolección.

Todos los pacientes registrados con complicaciones de cualquier tipo fueron clasificados en ocho categorías y se reportó el número de complicaciones por categoría en cada uno de los grupos. La comparación entre los grupos antes y después de la implementación de la estrategia fue analizada mediante el uso del programa SPSS versión 19.0 utilizando 


\begin{tabular}{lccc}
\multicolumn{4}{c}{ Cuadro I. Características basales. } \\
& Grupo I (255) & Grupo II (280) \\
Variable & n (\%) & n (\%) & p \\
\hline Edad en años (media \pm DE) & $40.3 \pm 22.1$ & $40.5 \pm 22.3$ & 0.9 \\
Hombres & $122(47.84)$ & $121(43.22)$ & \\
Mujeres & $133(52.16)$ & $159(56.78)$ & 0.28 \\
Cirugía electiva & $204(80.00)$ & $246(87.86)$ & 0.004 \\
Cirugía de urgencia & $51(20.00)$ & $34(12.14)$ & 0.01 \\
Cirugía mayor & $54(21.18)$ & $54(19.29)$ & 0.57 \\
Cirugía menor & $201(78.82)$ & $226(80.71)$ & 0.9 \\
Cirugías no complicadas & $226(88.63)$ & $268(95.7)$ & 0.34 \\
Cirugía complicada & $29(11.37)$ & $12(4.3)$ & 0.003 \\
ASA $>$ II & $101(39.60)$ & $135(48.21)$ & 0.08
\end{tabular}

Cuadro II. Se describen los desenlaces que se midieron si al aplicar la lista de verificación de cirugía segura fue funcional para control en los servicios de calidad y seguridad en el paciente quirúrgico.

\begin{tabular}{|c|c|c|c|}
\hline Variable & $\begin{array}{c}\text { Grupo I (255) } \\
\text { n }(\%)\end{array}$ & $\begin{array}{c}\text { Grupo II (280) } \\
\text { n (\%) }\end{array}$ & $\mathbf{p}$ \\
\hline Complicados & $29(11.4)$ & $12(4.3)$ & 0.003 \\
\hline Muerte quirúrgica & $2(0.78)$ & $1(0.35)$ & 0.45 \\
\hline Hemorragia mayor & $12(4.7)$ & $4(1.4)$ & 0.02 \\
\hline Infección postquirúrgica & $8(3.1)$ & $2(0.7)$ & 0.03 \\
\hline $\begin{array}{l}\text { Reacción adversa a } \\
\text { medicamentos }\end{array}$ & $2(0.78)$ & $1(0.35)$ & 0.22 \\
\hline Iatropatogenia & $3(1.2)$ & $2(0.7)$ & 0.45 \\
\hline $\begin{array}{l}\text { Complicaciones } \\
\text { anestésicas }\end{array}$ & $3(1.2)$ & $1(0.4)$ & 0.27 \\
\hline Complicaciones diversas & $5(2.0)$ & $1(0.4)$ & 0.08 \\
\hline
\end{tabular}

la prueba exacta de Fisher, así como la Chi cuadrada $\left(\chi^{2}\right)$.

El análisis se realizó para evaluar el efecto de la lista de verificación sobre la mortalidad y complicaciones en los individuos sometidos a intervención quirúrgica.

Se recolectaron de forma retrospectiva los datos de los sujetos intervenidos quirúrgicamente en el periodo comprendido del primero de enero al 31 de junio de 2010; y se prosiguió con los pacientes de 2012 que tuvieron complicaciones postquirúrgicas, incluyendo muerte, hasta los 30 días posteriores al procedimiento quirúrgico. Se compararon las características de la población entre los grupos I y II (Cuadro I).

De los individuos sometidos a cirugía, del grupo I, con 255 sujetos (antes de aplicar la lista de verificación), 122 fueron del sexo masculino y 133 del femenino; 204 se sometieron a cirugía electiva y 51 a cirugía de urgencia. En el grupo II se incluyeron un total de 280 personas, después de aplicar la lista de verificación; 121 correspondió al sexo masculino y 159 al femenino. La mayoría de los pacientes se sometieron a cirugía electiva (246); sólo 34 pacientes fueron cirugías de urgencia.

\section{RESULTADOS}

En el grupo II (al que se aplicó la estrategia de seguridad) hubo complicaciones en 12 pacientes, que representan el $4.3 \%$, en contraste con el grupo I (sin la estrategia), con un total de 29 pacientes con complicaciones, que representan el $11.4 \%$; al aplicar la prueba estadística de $\chi^{2}$, se demuestra que sí hubo significancia estadística $(p=0.003)$, con un riesgo relativo (RR) de 0.377; es decir, que la estrategia nos protege de cualquier tipo de complicación prevenible en un $62.3 \%$ (Cuadro II).

En cuanto a muerte quirúrgica, con la aplicación de la estrategia se presentó una disminución de la mortalidad de 0.8 a $0.4 \%$, aunque al aplicar la prueba exacta de Fisher $(p=0.45)$ no se encontró diferencia estadísticamente significativa entre ambos grupos. El tipo de complicaciones en el que se encontró una mayor relevancia al aplicar la estrategia de seguridad fueron el de infección postquirúrgica y el de sangrado mayor a lo permisible.

La infección postquirúrgica tuvo significancia estadística debido a que disminuyó del 3.1\% en el grupo I a $0.7 \%$ en el grupo II ( $p=0.03)$, con un riesgo relativo de 0.228 , lo que traduce que al grupo al que se le aplicó la estrategia tuvo un factor protector de $77.2 \%$ (Cuadro II).

En las complicaciones por hemorragia mayor, también se encontró significancia estadística $(\mathrm{p}=0.02)$ con una reducción de $4.7 \%$ (grupo I) a 1.4\% (grupo II), con un riesgo relativo (RR) de 0.403 , lo que significa que la estrategia de seguridad protege en un 59.7\% (Cuadro II).

En lo referente a las complicaciones anestésicas $(p=0.27)$, reacciones adversas a 
medicamentos $(p=0.22)$, iatropatogenia $(p$ $=0.45)$ y complicaciones diversas $(p=0.08)$, no se encontró diferencia estadísticamente significativa (Cuadro II).

\section{DISCUSIÓN}

En este estudio se observó que la implementación en el Hospital Naval de Mazatlán de la lista de verificación de cirugía segura propuesta por la OMS se asocia a una reducción de las complicaciones postquirúrgicas de 11.4\% a $4.3 \%$ ( $p=0.02$ ), así como a la reducción de la mortalidad postquirúrgica de 0.8 a $0.4 \%$, aunque ésta no fue de relevancia estadística $(p=0.45)$. Esto es similar a los trabajos de de Vries, Haynes y Weiser, ${ }^{6-8}$ realizados a nivel internacional, de tipo multicéntrico, los cuales demostraron que las tasas de morbilidad y mortalidad se reducen de manera significativa; los estudios no variaron en los hospitales incluidos en esta investigación, y en cuanto al número total de complicaciones por 100 pacientes, disminuyó de 27.3 a $16.7 \%$, con una reducción de riesgo de 10.6, según de Vries (2010). ${ }^{6}$ En otro de los trabajos de trascendencia, Haynes y Weisner $(2010)^{7,8}$ indicaron que la tasa de mortalidad se redujo de 1.5 a $0.8 \%$ ( $p=0.003)$, y las complicaciones quirúrgicas disminuyeron de 11 a 7\%; todas estas reducciones en morbilidad y mortalidad se asocian fuertemente a la aplicación de la lista de verificación de cirugía segura.

El reto es involucrar activamente a todo el personal que interviene en la atención del paciente para desarrollar la cultura de la seguridad del mismo. Es necesaria la participación de los directivos, jefes de servicio, supervisoras, médicos y enfermeras para lograr el objetivo final, que es la seguridad del enfermo. El porcentaje de cumplimiento antes del proyecto de mejora era de 54.8\%, y tras la evaluación del cumplimiento hubo un incremento significativo que osciló entre 75.9 y $94.6 \%{ }^{9}$

La aplicación correcta de la lista de verificación como parte del protocolo establecido por las metas internacionales de seguridad del paciente logra detectar fallas en la atención médica, y en consecuencia, previene en 50\% la frecuencia de quejas o demandas relacionadas con la atención médica. ${ }^{10}$

\section{CONCLUSIÓN}

Se observó una reducción de más del 60\% de las complicaciones posteriormente a la aplicación de la lista de verificación de cirugía segura, lo que representa la misma efectividad al compararse con el resto de los estudios realizados en hospitales a nivel internacional.

Se pudo identificar a la infección postquirúrgica y la hemorragia mayor a lo permisible como las complicaciones más frecuentes en este hospital, las cuales, al implementar la estrategia de seguridad, se redujeron considerablemente. Esto nos ayudará a tomar medidas de mejora continua que beneficiarán tanto al paciente como a la propia institución, al disminuir gastos, previendo posibles complicaciones adversas y muerte quirúrgica.

La demostración objetiva de los beneficios que se obtienen con la implementación de la lista de verificación son claros; la utilización de este instrumento ha reducido de manera importante la morbilidad, las complicaciones y la mortalidad. Es necesario continuar construyendo el aseguramiento del paciente quirúrgico con la total adhesión e implementación de todas las barreras que reduzcan los riesgos y eventos adversos. La participación conjunta y corresponsable de los pacientes y profesionales de la salud, y la sistematización de todas las medidas, con plena conciencia y profesionalismo, conducirán a una mejor práctica médica.

\section{REFERENCIAS}

1. Haynes AB, Weiser TG, Berry WR, Lipsitz SR, Breizat AHS, Dellinger P, et al. A surgical safety checklist to reduce morbidity and mortality in a global population. N Engl J Med. 2009; 360: 491-499.

2. Organización Mundial de la Salud. Segundo reto internacional por la seguridad del paciente, La cirugía segura salva vidas. WHO/IER/PSP/2008 07.

3. Weiser TG, Regenbogen SE, Thompson KD, Haynes $A B$, Lipsitz SR, Berry WR, et al. An estimation of the global volume of surgery: a modelling strategy based on available data. Lancet. 2008; 372: 139-144.

4. Organización Mundial de la Salud. La Investigación en seguridad del paciente, mayor conocimiento para una atención más segura. WHO/IER/PSP/2008 02.

5. Soria-Aledo V, Da Silva Z, Saturno P, Grau-Polan M, Carrillo-Alcaraz A. Dificultades en la implantación del 
checklist en los quirófanos de cirugía. Cir Esp. 2012; 90: 180-185.

6. de Vries EN, Prins HA, Crolla RM, den Outer AJ, van Andel G, van Helden SH, et al. Effect of a comprehensive surgical safety system on patient outcomes. N Engl J Med. 2010; 363: 1928-1937.

7. de Vries EN, Ramrattan MA, Smorenburg SM, et al. The incidence and nature of in-hospital adverse events: a systematic review. Qual Saf Health Care. 2008; 17: 216-223.

8. Weiser TG, Haynes AB, Dziekan G, Berry WR, Lipsitz SR, Gawande AA. Effect of a 19-item surgical safety checklist during urgent operations in a global patient population. Ann Surg. 2010; 251: 976-980.

9. Hernández-García. Efectividad de una intervención para mejorar la calidad de la cumplimentación del listado de verificación de seguridad quirúrgica en un servicio de cirugía general. [Internet] Disponible en: http://dx.doi.org/10.1016/j.cali.2015.12.005

10. Campos CC, Hurtado LLM, Basurto KE, Zaldivar RE. Prevención de quejas y demandas al aplicar las metas internacionales de seguridad en el paciente quirúrgico. Cir Gen. 2011; 33: 170-174.

\section{Correspondencia:}

Elena López Gavito

Avenida Rafael Buelna Núm. 198. L319C.

Poli médica.

Fraccionamiento Hacienda Las Cruces, 82126, Mazatlán, Sinaloa.

Tel. 016699842999

E-mail: elopezgavito@gmail.com 